\title{
Polypeptoid Material as an Anchoring Material for Li-S Batteries
}

\author{
Deobrat Singh* and Rajeev Ahuja*
}

Cite This: ACS Appl. Energy Mater. 2021, 4, 13070-13076

Read Online

ABSTRACT: Nowadays, lithium-sulfur $(\mathrm{Li}-\mathrm{S})$ batteries have attracted considerable attention as a potential candidate for next-generation rechargeable batteries due to their high theoretical specific energy and environmental friendliness. One of the main problems with $\mathrm{Li}-\mathrm{S}$ batteries is that the lithium polysulfides (LiPSs) easily decompose in the electrolyte which is known as the shuttle effect. Recently, the polypeptoid nanosheet crystal structure has been experimentally synthesized which is very useful for tremendous advances in soft material imaging as well as enabling to design biomimetic nanomaterials. Due to the very interesting properties of the polypeptoid material, we have investigated the electronic structure and charge-transfer mechanism for the lithium-sulfur batteries for the cathode material. The calculated adsorption energies of LiPSs on the surface of the polypeptoid material are in the range of -4.41 to -4.64 and $-0.91 \mathrm{eV}$ for the sulfur clusters. Also, the adsorption energies between the interaction of LiPSs and electrolytes (DME and DOL) are 0.75-0.89 eV. It means that the polypeptoid material could suppress the shuttle effect of LiPSs and significantly enhance the cycling performance of $\mathrm{Li}-\mathrm{S}$ batteries. From these investigated results, the polypeptoid material will be a promising anchoring material for $\mathrm{Li}-\mathrm{S}$ batteries.

KEYWORDS: polypeptoid material, electronic properties, charge-transfer mechanism, binding of lithium polysulfides, Li-S battery

\section{INTRODUCTION}

With the growing population and economic development, the demand for energy technology for portable energy storage in mobile applications, for example, portable/wearable electronics and electric vehicles, has attracted great attention these days. ${ }^{1-3}$ Energy-storage systems based on batteries are an important technology for stabilizing renewable energy use and enhancing energy security ${ }^{4}$ because batteries are the integrated parts of electric vehicles, laptop computers, and cell phones. ${ }^{5}$ Lithiumsulfur ( $\mathrm{Li}-\mathrm{S}$ ) batteries display better candidates for highperformance energy-storage technology because of the high theoretical specific capacity of $1675 \mathrm{~mA} \mathrm{~h} \mathrm{~g}^{-1}$, a high specific energy of $2600 \mathrm{~W} \mathrm{~h} \mathrm{~kg}^{-1}$ among the rechargeable batteries, and also low cost, environmentally friendly, and abundance in nature of sulfur which fabricates a commercially competitive material and is suitable for large-scale production. ${ }^{6-9}$ However, there are some challenges that still hinder their commercial potential such as (i) a very poor conductivity of sulfur, (ii) the critical potential vanishing occurs due to the shuttle effect of the polysulfide intermediates, ${ }^{10-13}$ and (iii) the large volume expansion due to the density difference of lithium and sulfur. ${ }^{14}$

To overcome these issues, several cathode materials have been studied to enhance the performance of batteries. ${ }^{15}$ Until date, significant efforts have been focused to design a carbonsulfur composite cathode to reduce the shuttle effect, but it remains challenging to suppress the migration and dissolution of polysulfides in the organic electrolyte. ${ }^{11,16-22}$ Recently, the significant modification of separators using different types of porous materials such as porous carbon ${ }^{23,24}$ and other types of porous materials, conducting polymers, ${ }^{25,26}$ metal-organic frameworks (MOFs), ${ }^{27}$ or $2 \mathrm{D}$ materials ${ }^{28,29}$ has been displayed as an effective way to improve the cycle performance of $\mathrm{Li}-\mathrm{S}$ batteries, in which the modified separator can impressively suppress polysulfide migration to the anodes. ${ }^{3}$ The reported literature suggests that the characteristics of the materials added such as the separator, electrolyte, interlayer, anode, and cathode in the separators are important factors in deciding the performance of the battery.

It was reported that the poly(leucine-lysine)-based peptide significantly suppresses the sulfur loss in the electrolyte and also proposes that it is very useful for potential applications in $\mathrm{Li}-\mathrm{S}$ batteries as a coating material. ${ }^{30}$ The polypeptoid material contains carbon $(\mathrm{C})$, oxygen $(\mathrm{O})$, and nitrogen $(\mathrm{N})$ atoms and benzene groups. Due to the effect of electronegativity, the atoms generate polar and nonpolar bonds between $\mathrm{C}-\mathrm{O}$ and $\mathrm{C}-\mathrm{N}$. Also, the benzene ring has a large electron cloud density that appears from a conjugated $\pi$-orbital electron which interacts with LiPSs and it could be the most active interaction site to inhibit the loss of polysulfides. Therefore, such types of materials

Received: September 1, 2021

Accepted: October 18, 2021

Published: October 29, 2021 


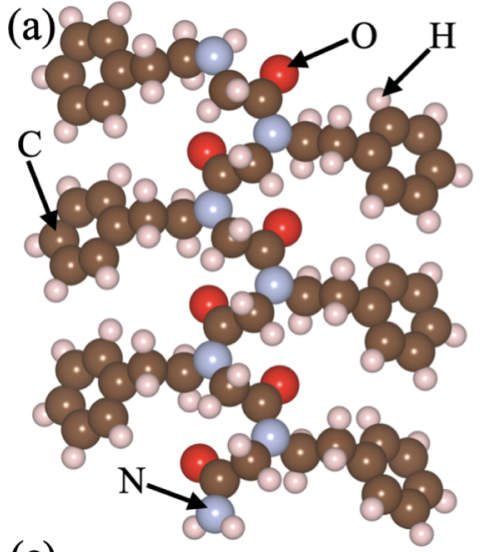

(c)

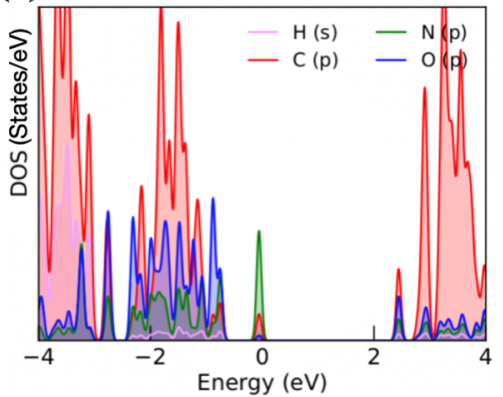

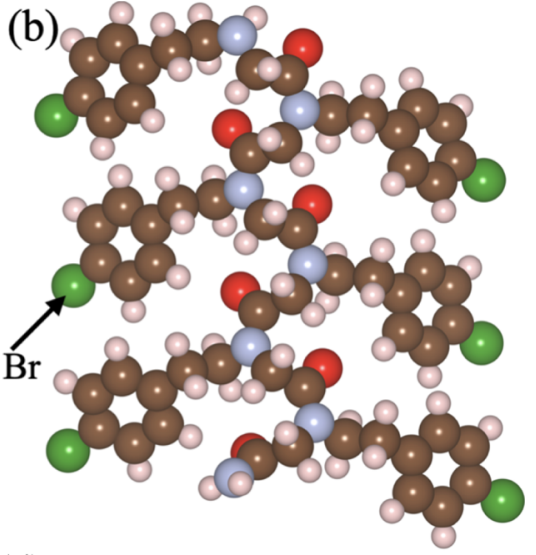

(d)

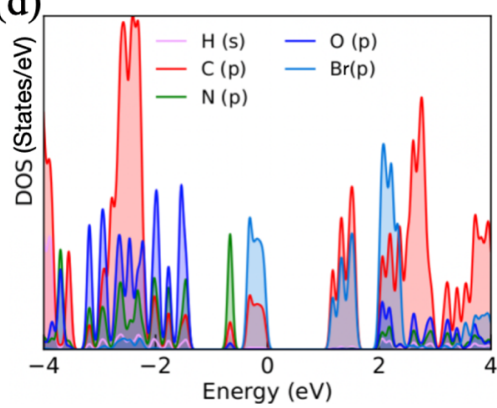

Figure 1. Chemical structures of (a) Nte4-Npe6 and (b) Nte4-N4Brpe6 polypeptoid materials. The carbon, oxygen, nitrogen, hydrogen, and bromine atoms are represented by brown, red, blue gray, light pink, and green, respectively. Projected density of states of (c) Nte4-Npe6 and (d) Nte4-N4Brpe6 polypeptoid materials.

could be better candidates for $\mathrm{Li}-\mathrm{S}$ batteries. In addition, a $2 \mathrm{D}$ polypeptoid nanosheet has been recently successfully synthesized. ${ }^{31}$ Motivated by these abovementioned studies, we have investigated the thermal stability at $300 \mathrm{~K}$ using ab initio molecular dynamics (AIMD) simulations and it maintained the structural configuration for $10 \mathrm{ps}$ and no breaking of bonds between the atoms confirmed the structural stability. In the present work, we have proposed the newly synthesized organic polypeptoid material structure for $\mathrm{Li}-\mathrm{S}$ batteries for cathode materials which are investigated by density functional theory calculations. The physical and chemical interactions of $\mathrm{Li}_{2} \mathrm{~S}_{x}(x=$ $1,2,4,6$, and 8$)$ and $S_{8}$ clusters with the polypeptoid material are investigated. Furthermore, we compute the nature of bonding and charge transfer between $\mathrm{Li}_{2} \mathrm{~S}_{x}$ and polypeptoid material. We have also investigated the diffusion and decomposition energy barrier of $\mathrm{Li}_{2} \mathrm{~S}$ which is lower than most of the materials used in $\mathrm{Li}-\mathrm{S}$ batteries. We found that these finding results such as strong binding energy between $\mathrm{Li}_{2} \mathrm{~S}_{x}$, the lower energy barrier of the polypeptoid material ensuring excellent materials for $\mathrm{Li}-\mathrm{S}$ batteries. $^{32}$

\section{COMPUTATIONAL METHODS}

The electronic structure calculations are based on the DFT approach using VASP code. ${ }^{33-35}$ To describe the core electrons and electronion interactions, the projector-augmented-wave pseudopotentials were used. $^{36}$ The exchange-correlation energy was described via the Perdew, Burke and Ernzerhof functional within generalized gradient approximation. ${ }^{37}$ The energy cutoff of $500 \mathrm{eV}$ has been used for the plane-wave basis set, and $\Gamma$-point sampling was used. To prevent the physical interaction between the periodic images, we have taken $15 \AA$ in each direction. We have used the DFT-D3 procedure in order to include the van der Waals interaction. ${ }^{38,39}$ For the chemical structure visualizations and plots, we have used VESTA software. ${ }^{40}$ During the calculations for self-consistent and electronic density of states, we have used the Gaussian broadening of $0.06 \mathrm{eV}$. For structural optimizations, a combination of conjugate-gradient algorithm and a quasi-Newton force minimization was used. ${ }^{41}$ During the calculations, all the atoms were allowed to fully relax until forces acting on atoms were less than $0.005 \mathrm{eV} / \AA$. To determine the energy barrier, we have used the climbing image nudged elastic band method that is implemented in the VASP-VTST tools. ${ }^{42,43}$ We have used the AIMD simulations to check the thermal stability of the polypeptoid material at room temperature. The NVT ensemble has been used for 10 ps with a time step of $2 \mathrm{fs}$, and the considered temperature was controlled by the Nosé-Hoover method. ${ }^{44}$ The Bader charge analysis ${ }^{45}$ has been used to calculate the charge transfer between the LiPS species and substrate.

\section{RESULTS AND DISCUSSION}

Structural and Electronic Properties. The optimized structures of polypeptoid material with different configurations, that is, Nte4-Npe6 (conf.-1) and Nte4-N4Brpe6 with Br atoms (conf.-2), are shown in Figure 1. Here, poly $(\mathrm{N}-2-(2-(2-$ methoxyethoxy)ethoxy)ethylglycine) is represented by Nte and $\mathrm{N}$-(2-phenylethyl)glycine is represented by Npe. The considered polypeptoids Nte4-Npe6 and Nte4-N4Brpe6 are almost similar, but in the case of polypeptoids Nte4-Npe6, each benzene ring is attached by one hydrogen $(\mathrm{H})$ atom, while in the case of polypeptoids Nte4-N4Brpe6 configuration, each benzene ring is connected by bromine $(\mathrm{Br})$ atoms. Here, the electronegativity of the $\mathrm{Br}$ atom is larger than the $\mathrm{H}$ atom; therefore, it could affect the physical and chemical properties of materials. The optimized bond lengths between $\mathrm{C}-\mathrm{C}, \mathrm{C}-\mathrm{O}$, $\mathrm{C}-\mathrm{N}, \mathrm{C}-\mathrm{H}, \mathrm{N}-\mathrm{H}$, and $\mathrm{C}-\mathrm{Br}$ are $1.40 \AA$ in a hexagonal ring (1.54 $\AA$ in other places), 1.23 and $1.37 \AA$ when $\mathrm{C}$ is attached with an $\mathrm{O}$ atom ( $1.45 \AA$ when $\mathrm{C}$ is attached with a $\mathrm{H}$ atom), and 1.10, 1.01 , and $1.53 \AA$ in conf.- 1 and conf.- 2 which is well consistent with the previous literature. ${ }^{31}$ Moreover, we have investigated the AIMD simulations to check the thermal stability of the 
(a)

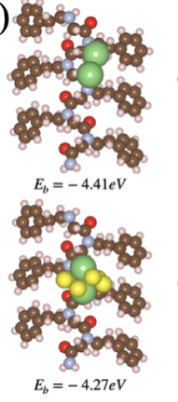

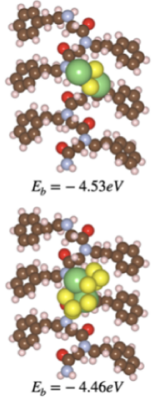

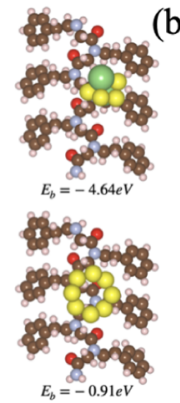

(b)

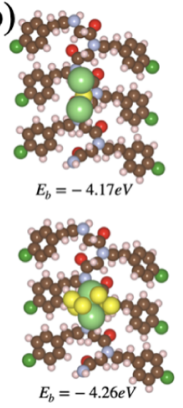

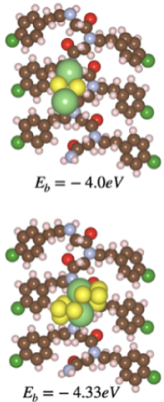

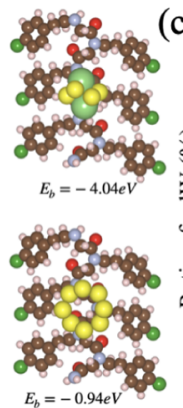

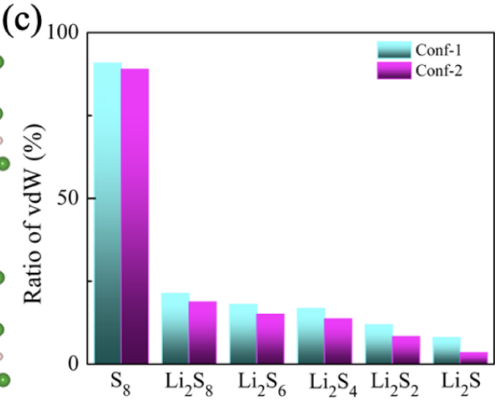

Figure 2. Binding strength of $\mathrm{Li}-\mathrm{S}$ composites on the surface of the polypeptoid material of (a) conf.-1 and (b) conf.-2. (c) Ratio for vdW interaction for the polypeptoid material of conf.-1 and conf.-2 at different lithation stages.

polypeptoid material. The final structures of polypeptoid material are depicted in Figures S1 and S2 (see the Supporting Information). The AIMD simulations have been performed at room temperature for $10 \mathrm{ps}$ and it was seen that there is no structure distortion and no bond breaking inside the polypeptoid material. It means that the polypeptoid material has good thermal stability.

Furthermore, to better understand the electronic properties of the polypeptoid material, we have investigated the density of states (DOS) (see Figure 1c,d). From the DOS, the highestoccupied molecular orbital (HOMO) and lowest-unoccupied molecular orbital (LUMO) are well-separated, which means that both configurations displayed the semiconducting behavior. The HOMO-LUMO gap in the case of conf.- 1 and conf.- 2 is 2.47 and $1.25 \mathrm{eV}$, respectively. Also, the HOMO is mostly originated by $\mathrm{N}$ p-orbitals, while the LUMO is mostly contributed by $\mathrm{p}$ orbitals of $\mathrm{C}$ and $\mathrm{O}$ atoms. It means that these states are dominating near the Fermi level (see Figure 2a), in conf.-1. While in the case of conf.-2, the HOMO is formed by p-orbitals of both $\mathrm{Br}$ and a less part originated from $\mathrm{C} \mathrm{p}$-orbitals, and the LUMO is mostly originated by p-orbitals of $\mathrm{Br}$ and $\mathrm{C}$ atoms. Furthermore, from the Bader charge analysis, the atomic charges are arranged as per requirement. The $\mathrm{N}$ atom gains $1.10 \mathrm{e}$, the $\mathrm{C}$ atom donates $0.14 \mathrm{e}, \mathrm{O}$ atoms gain $1.13 \mathrm{e}$, and the $\mathrm{H}$ atom donates $0.06 \mathrm{e}$ to the surrounding atoms in conf.-1. Similarly, in the case of conf.-2, the $\mathrm{N}, \mathrm{C}, \mathrm{O}, \mathrm{H}$, and $\mathrm{Br}$ atoms 1.13 e (gains), 0.11 e (donates), 1.13 e (gains), 0.07 e (donates), and $0.71 \mathrm{e}$ (donates) to the surrounding atoms, respectively. More importantly, due to the presence of oxygen and nitrogen atoms in the middle part of the polypeptoid material, therefore, it would enhance the performance of the $\mathrm{Li}-\mathrm{S}$ battery by anchoring a polar lithium polysulfide intermediate.

Adsorption and Charge-Transfer Mechanism. One of the main difficulties with $\mathrm{Li}-\mathrm{S}$ batteries is that the intermediate LiPSs easily dissolve into the electrolyte which reduces the performance of the $\mathrm{Li}-\mathrm{S}$ batteries. To prevent the shuttle effect, that is, dissolution of LiPS species into the electrolyte, it is necessary that the binding energy of LiPS species with the anchoring material should be larger than that of the organic electrolyte molecules. The optimized molecular structures of LiPSs and organic electrolytes are presented in Figure S3 (see the Supporting Information). The binding energy $\left(E_{\mathrm{b}}\right)$ of LiPSs on the surface of the polypeptoid material is given as

$$
E_{\mathrm{b}}=E_{(\text {(substrate }+\mathrm{LiPSs})}-E_{\text {substrate }}-E_{\mathrm{LiPS}}
$$

where $E_{(\text {substrate+LiPSs) }}, E_{\text {substrate }}$ and $E_{\text {LiPSs }}$ represent the total energy of the substrate with LiPSs, substrate, and isolated LiPS species, respectively. Using eq 1 , we have investigated the binding energy of LiPSs and $S_{8}$ species on the polypeptoid material. During the charging and discharging process, $S$ containing clusters such as $\mathrm{Li}_{2} \mathrm{~S}_{x}$ (i.e., $x=1,2,4,6$, and 8 ) and $\mathrm{S}_{8}$ are generated. Initially, we have considered different orientations and different positions in which Figure 2 displayed the fully optimized with the lowest energy configuration for both cases. Particularly, for these types of adsorption systems, previous investigations have demonstrated that the bond appears through the coordinated effect of vdW and covalent bonding. ${ }^{46}$ First, we have systematically investigated the ratio of $\mathrm{vdW}$ interactions to see the strength of chemical and physical adsorption influence of anchoring effects. Here, the vdW interaction ratio is as follows

$$
R_{\mathrm{vdW}}(\%)=\left(\frac{E_{\mathrm{b}}^{\mathrm{PBE}+\mathrm{vdW}}-E_{\mathrm{b}}^{\mathrm{PBE}}}{E_{\mathrm{b}}^{\mathrm{PBE}+\mathrm{vdW}}}\right) 100
$$

where $E_{\mathrm{b}}^{\mathrm{PBE}}$ and $E_{\mathrm{b}}^{\mathrm{PBE}+\mathrm{vdW}}$ are the terms of $E_{\mathrm{b}}$ which comes from the standard PBE functional and PBE functional with the addition of the $\mathrm{vdW}$ term. Figure 2 displays the strength of $\mathrm{vdW}$ interactions provided significantly and clearly for two different configurations of the electrodes. The chemical and $\mathrm{vdW}$ interactions play a significant role in the case of de-lithiation from $\mathrm{Li}_{2} \mathrm{~S}$ to $\mathrm{Li}_{2} \mathrm{~S}_{8}$, in which the strength of the vdW interaction to the binding energies conclusively increases and reaches up to 21.35 and $19 \%$ in conf.- 1 and conf.-2, respectively. While the ratio of the vdW interaction for species $\mathrm{S}_{8}$ has almost reached 90\% (see Figure 2c). All the lowest adsorption configurations with the top view of $\mathrm{Li}_{2} \mathrm{~S}_{x}$ and $\mathrm{S}_{8}$ species are depicted in Figure $2 \mathrm{a}, \mathrm{b}$ for conf.- 1 and conf.-2, respectively. Additionally, the side view of these two configurations is presented in Figures $\mathrm{S} 4$ and S5 (see the Supporting Information). It was seen that the $S_{8}$ species lies parallel to the top of the polypeptoid sheet and the vertical distance of $2.99 \AA / 3.29 \AA$ for conf.-1/conf.- 2 of the polypeptoid material, respectively. From the Bader charge analysis, a relatively small amount of charge transfer $(0.074 \mathrm{e} /$ $0.02 \mathrm{e}$ for conf.-1/conf.-2) has been found. The less amount of charge transfer indicates the weak chemical interaction between the $S_{8}$ species and polypeptoid surface which is also confirmed by the relatively lower binding strength (see Figure 2a,b).

From Figure 2a,b, we can clearly see that the positions and orientations of adsorbed species, that is, LiPSs and $\mathrm{S}_{8}$ clusters on the surface of the polypeptoid, are slightly different. Because the electronegativity of the $\mathrm{Br}$ atom is larger than the $\mathrm{H}$ atom attached with benzene rings in conf.- 1 and conf.-2, therefore, it affected the physical/chemical interactions of adsorbed species on these two configurations. Figure 2a shows that the other adsorption configurations in which $\mathrm{Li}_{2} \mathrm{~S}_{8}$ species prefers relatively lying near to the substrate and both $\mathrm{Li}$ atoms lie toward the polypeptoid surface in both configurations. Similar to 
$\mathrm{Li}_{2} \mathrm{~S}_{8}$ species, the $\mathrm{Li}_{2} \mathrm{~S}_{6}$ species also prefer the same orientation, and the vertical height between the species and substrate is almost equal. While both $\mathrm{Li}$ atoms are parallel to the surface in the case of conf.- 1 and conf.- 2 , the one $\mathrm{Li}$ atom is slightly lower than other $\mathrm{Li}$ atoms in the adsorbed $\mathrm{Li}_{2} \mathrm{~S}_{6}$ species on the surface of the polypeptoid. In contrast, the lower-order species $\mathrm{Li}_{2} \mathrm{~S}_{x}(x$ $=1,2$, and 4) prefer to form chemical bonding of one $\mathrm{Li}$ atom to $\mathrm{N}$ atom from the polypeptoid surface in the case of conf.-1, whereas one $\mathrm{Li}$ atom of $\mathrm{Li}_{2} \mathrm{~S}_{2}$ species forms chemical bonding to the polypeptoid surface, and the other two only have a chemical interaction with the substrate. Additionally, the lower-order species $\mathrm{Li}_{2} \mathrm{~S}_{x}(x=1,2$, and 4$)$ have relatively lower vertical height between the species and substrate, and also, it has higher charge transfer between them (see Table 1). As the discharge process

Table 1. Optimized Structural Parameters Such as Vertical Distance between the Polypeptoid Material and LiPS Species $^{a}$

\begin{tabular}{clcccccc} 
& & $\mathrm{S}_{8}$ & $\mathrm{Li}_{2} \mathrm{~S}_{8}$ & $\mathrm{Li}_{2} \mathrm{~S}_{6}$ & $\mathrm{Li}_{2} \mathrm{~S}_{4}$ & $\mathrm{Li}_{2} \mathrm{~S}_{2}$ & $\mathrm{Li}_{2} \mathrm{~S}$ \\
\multirow{3}{*}{ conf-1 } & $d_{\text {sheet-LiPS }}$ & 2.99 & 2.61 & 2.66 & 1.95 & 1.79 & 1.90 \\
& $Q$ & 0.07 & 0.13 & 0.21 & 0.31 & 0.54 & 0.61 \\
conf-2 & $d_{\text {sheet-LiPSs }}$ & 3.29 & 2.65 & 2.85 & 3.02 & 2.07 & 2.17 \\
& $Q$ & 0.02 & 0.09 & 0.18 & 0.28 & 0.45 & 0.48
\end{tabular}

${ }^{a}$ Charge-transfer values between the polypeptoid material and LiPS species. The positive values of charge represent the transfer of LiPS species to the polypeptoid surface.

proceeds, more and more electrons are transferred from the LiPSs species to the polypeptoid substrate, which shows that the strength of chemical interactions increases during the lithiation process. The positive values of the charge transfer display the polypeptoid that gains electrons from the LiPSs and $S_{8}$ species, as presented in Table 1 . In both cases conf.-1 and conf.-2, electrons are transferred from LiPSs and $S_{8}$ species to the polypeptoid surface. It was seen that the charge-transfer values in conf.- 1 are slightly larger than those of conf.-2, which reflects the binding strength between the LiPSs and $S_{8}$ species and polypeptoid surface. Also, a less amount of charge transfer refers to the relatively low chemical interactions. Apart from this, the lower-order S-containing species in LiPSs have higher charge transfer as compared to the higher-order S-containing species in LiPSs (see Table 1). From these investigations, we can see that when $\mathrm{Br}$ atoms are attached with each benzene ring (conf.-2), then the binding strength of LiPSs is slightly decreased as compared to $\mathrm{H}$-connected benzene rings (conf.-1). Generally, $\mathrm{Li}_{2} \mathrm{~S}_{x}$ clusters are also nicely maintained during adsorption, indicating that the polypeptoid sheet is a suitable candidate for $\mathrm{Li}-\mathrm{S}$ batteries as a cathode anchoring material.

Furthermore, to analyze the anchoring performance of the polypeptoid material, we have systematically investigated the binding energies of LiPSs on the surface of the polypeptoid material and the corresponding results are presented in Figure 3a. It was reported that the suitable solvent for the $\mathrm{Li}-\mathrm{S}$ cell electrolytes is limited such as dimethyl ether (DME) and 1,3dioxolane (DOL) used commonly. ${ }^{47,48}$ It was seen that both DME and DOL electrolytes lead to synergistic effects on the retention capacity and specific capacity of sulfur as compared to the solvent alone. ${ }^{49}$ Because of this, we have investigated the binding energies of commonly used electrolytes (DOL and DME) with LiPS species. The considered electrolyte is the liquid phase in which $S_{8}$ species is not soluble. ${ }^{50}$ The fully optimized structures of isolated LiPSs, as well as $\mathrm{S}_{8}$ and electrolyte (DOL and DME), are presented in Figure S3 in the Supporting Information. Also, the lowest energy configuration structures of LiPSs with an electrolyte (DOL and DME) are presented in Figure S6 in the Supporting Information. From Figure 3a, we can see that the polypeptoid material shows better performance for all lithium polysulfides.

Due to the presence of only $S$ atoms in $S_{8}$ species, it has no polarity. Therefore, the $E_{\mathrm{b}}$ of $S_{8}$ for both the configurations is nearly equal and relatively very less as compared to other
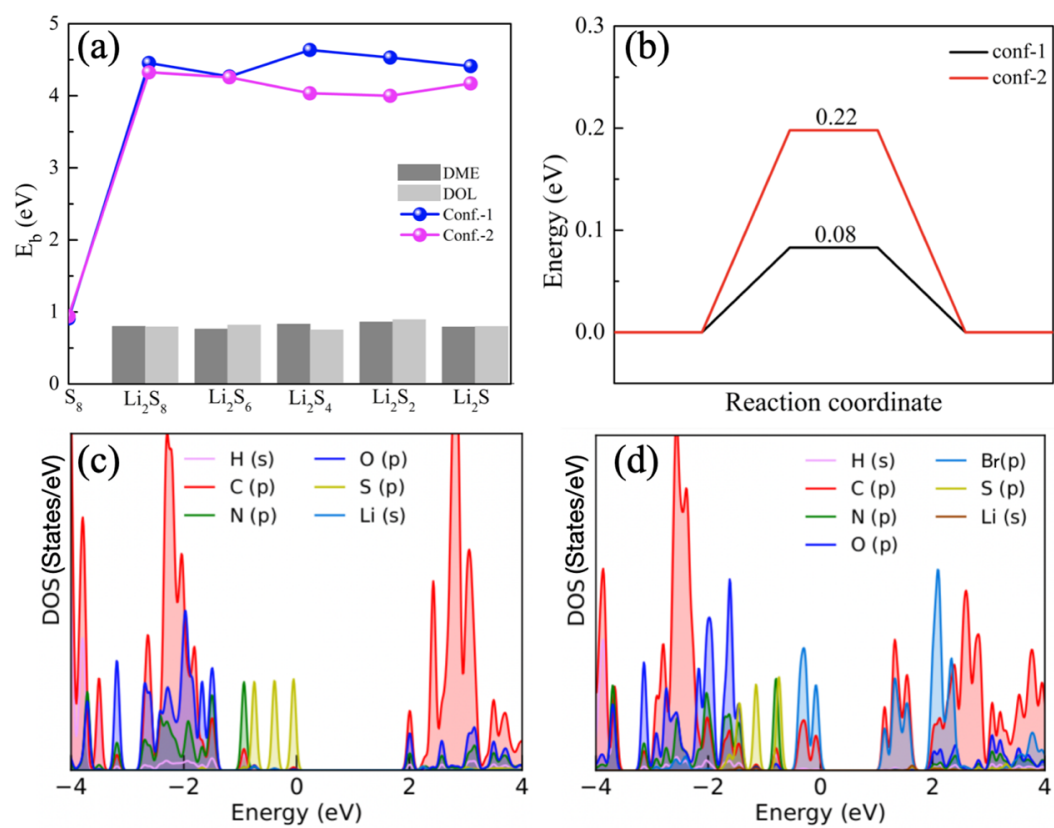

Figure 3. (a) Binding strength of LiPSs and $S_{8}$ clusters on the surface of the polypeptoid material and the corresponding binding energies of LiPSs with electrolytes for comparison. (b) Calculated energy barriers of $\mathrm{Li}_{2} \mathrm{~S}$ diffusion on the surface of the polypeptoid material. (c,d) Projected DOS polypeptoid material. 

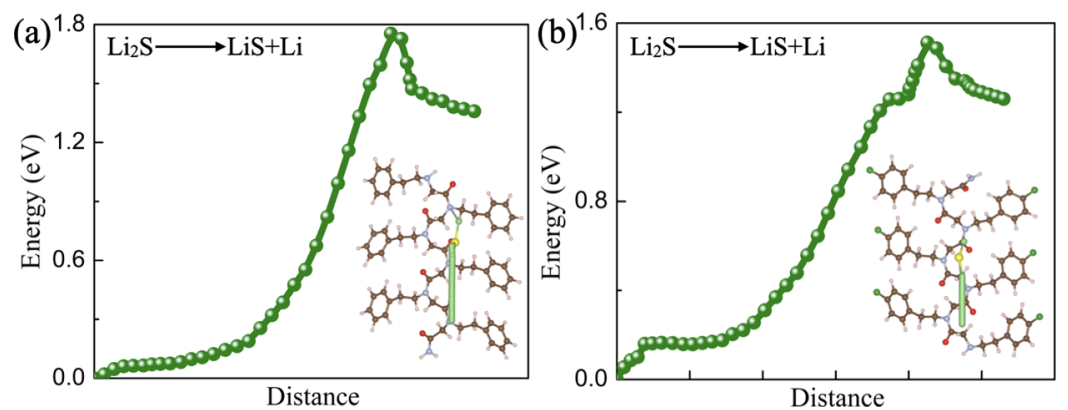

Figure 4. Diffusion process of decomposition of $\mathrm{Li}_{2} \mathrm{~S}$ species and energy curve on the surface of the polypeptoid material for (a) conf.- 1 and (b) conf.-2.

adsorbed species. ${ }^{51}$ Also, due to no polarity in $S_{8}$ species trying to lie in a parallel orientation to the surface, it has a very small charge transfer between them. Whenever the top surface contains $\mathrm{O}, \mathrm{F}, \mathrm{Cl}$, and $\mathrm{N}$ atoms (i.e., generally higher electronegativity as compared to the rest of the atoms) in the materials, then such types of materials could capture more effectively the polar LiPS species than the rest of the layered materials. ${ }^{50,51}$ Due to the presence of $\mathrm{O}$ and $\mathrm{N}$ at the middle of the Nte4-Npe6 and Nte4-N4Brpe6 polypeptoid material, it has more strength to capture the polar LiPS species. The calculated binding energies of Nte4-Npe6 and Nte4-N4Brpe6 polypeptoid material are mentioned (see Figure $3 \mathrm{a}$ ). The $E_{\mathrm{b}}$ is varying from 0.91 to 4.64 and 0.94 to $4.33 \mathrm{eV}$ for conf.- 1 and conf.-2, respectively, and $0.75-0.89 \mathrm{eV}$ for both electrolytes. The optimized interactions between the electrolytes and LiPSs species are presented in Figure S6 (see the Supporting Information). The binding energies between electrolytes and LiPSs species are lower than the corresponding binding energy values between the polypeptoid material and LiPS species which suggest that the LiPS species strongly absorb on the polypeptoid material rather than dissolve into the electrolytes. Furthermore, the binding energies over the polypeptoid material are stronger than most of the studied 2D-layered materials. ${ }^{51-53}$ It means that the polypeptoid material is firmly anchored to all sulfurcontaining species. From the abovementioned descriptions, both configurations of the polypeptoid material have a stronger affinity to bind the sulfur-containing clusters that suppress the shuttle effect which as a result improves the overall performance of $\mathrm{Li}-\mathrm{S}$ batteries.

Also for anchoring material, the surface diffusion properties are an important parameter that will be affected by the growth and distribution of sulfide species at the electrode, whereby the optimized balance between the adsorption of sulfur-containing clusters and surface diffusion properties display a significant role in $\mathrm{Li}-\mathrm{S}$ battery performance. ${ }^{54}$ During discharging case, the cathode of $\mathrm{Li}-\mathrm{S}$ batteries is defined as

$$
\mathrm{S}_{8}+16 \mathrm{Li}^{+}+16 \mathrm{e}^{-} \rightarrow 8 \mathrm{Li}_{2} \mathrm{~S}
$$

That is why we have considered $\mathrm{Li}_{2} \mathrm{~S}$ species to compute the energy barrier toward the particular diffusion pathway for conf.1 and conf.-2. Initially, we have optimized the vertical distance to get the minimum energy configuration of polypeptoid materials with $\mathrm{Li}_{2} \mathrm{~S}$ species. Also, we have checked the two most favorable positions of $\mathrm{Li}_{2} \mathrm{~S}$ species with minimum energy configurations; after that, we have decided the diffusion direction to get the activation energy. The activation energy is calculated by the climbing image-nudged elastic band method. Figure $3 \mathrm{~b}$ displays the diffusion energy barriers of conf.- 1 and conf.- 2 of the polypeptoid material and the corresponding energy barriers are
0.08 and $0.22 \mathrm{eV}$, respectively. From the energy barrier, conf.- 1 has a lower value for the diffusion of $\mathrm{Li}_{2} \mathrm{~S}$ species on the surface of the polypeptoid material. From the Arrhenius equation, the diffusion constant $(D)$ is directly proportional to the $\exp \left(-E_{\mathrm{a}} /\right.$ $\left.k_{\mathrm{B}} T\right) .^{55}$ In this equation, $E_{\mathrm{a}}, k_{\mathrm{B}}$, and $T$ represent the energy barrier, Boltzmann's constant, and absolute temperature, respectively. Here, we have estimated the diffusion constant (D) of $\mathrm{Li}_{2} \mathrm{~S}$ at room temperature which is $\sim 10^{6}$ times faster than nonconductive metal oxide (i.e., energy barrier lies between 0.45 and $1.22 \mathrm{eV})^{54}$ and 22 times faster than conductive layered materials (i.e., their energy barrier lies between 0.22 and 0.16 $\mathrm{eV}) .{ }^{50,55}$ It means that when it will be used as a cathode material in $\mathrm{Li}-\mathrm{S}$ batteries, then we expect that the polypeptoid material will give good cycling performance.

The good electrical conductivity of sulfur cathode host materials is also affecting the operability and performance of the $\mathrm{Li}-\mathrm{S}$ batteries which is essential to significantly govern the performance of $\mathrm{Li}-\mathrm{S}$ batteries. Therefore, to understand the electrical conductivity, the DOS of $\mathrm{Li}_{2} \mathrm{~S}$ adsorbed on the surface of the polypeptoid material has been calculated. Figure $3 c, d$ represents the PDOS for conf.- 1 and conf.- 2 of the polypeptoid material after the adsorption of $\mathrm{Li}_{2} \mathrm{~S}$ species. From Figure $3 \mathrm{c}, \mathrm{d}$, the significantly enhanced electronic states near the $F_{F}$ are observed. Here, we found that the $\mathrm{Li}_{2} \mathrm{~S}$ cluster adsorbed on polypeptoid materials and then it reduces the HOMO-LUMO gap from 2.47 to $1.99 \mathrm{eV}$ for conf.-1 while 1.25 to $1.13 \mathrm{eV}$ for conf.-2. During the adsorption of $\mathrm{Li}_{2} \mathrm{~S}$ species on the surface of polypeptoid materials, the $\mathrm{Li}_{2} \mathrm{~S}$ species transfer 0.61 e and $0.48 \mathrm{e}$ to the surface in the case of conf.-1 and conf.-2, respectively, which is confirmed by Bader charge analysis (see Table 1). It means that the $\mathrm{S}$ atom from $\mathrm{Li}_{2} \mathrm{~S}$ species is strongly hybridized with $\mathrm{C}, \mathrm{N}$, and $\mathrm{O}$ atoms from the surface in both configurations. Also, it was seen that the Fermi level is shifted because the electronic states come from $S$ orbitals, that is, HOMO is mainly made by $\mathrm{S}$ orbitals from $\mathrm{Li}_{2} \mathrm{~S}$ species in the case of conf.- 1 . However, in the case of conf.-2, some of the electronic states appear around $-1 \mathrm{eV}$ in the HOMO due to the significant hybridization of the orbitals of $\mathrm{Li}_{2} \mathrm{~S}$ species and surface atoms. These hybridizations are responsible for enhancing the electrical conductivity in the considered materials. Due to the presence of an electronic state near the Fermi level, it enhances the electrical conductivity of the polypeptoid material after the adsorption of the $\mathrm{Li}_{2} \mathrm{~S}$ cluster that will significantly enhance the performance of $\mathrm{Li}-\mathrm{S}$ batteries.

It is well-known that the low-order sulfur-containing cluster $\mathrm{Li}_{2} \mathrm{~S}$ begins to decompose and oxidize to high-order sulfur clusters and finally form $S_{8}$ clusters during the charging process of $\mathrm{Li}-\mathrm{S}$ batteries. It is also well-known that catalysis of the decomposition of $\mathrm{Li}_{2} \mathrm{~S}$ clusters near the surface of the substrate is a vital step to appreciate high capacity, as well as Coulombic 
efficiency. ${ }^{56}$ Apart from this, the energy barrier of the decomposition $\mathrm{Li}_{2} \mathrm{~S}$ species qualitatively agrees by measurement of voltage magnitude in experiments. ${ }^{56}$ In the present work, the decomposition process of $\mathrm{Li}_{2} \mathrm{~S}$ species from $\mathrm{Li}_{2} \mathrm{~S}$ molecules into $\mathrm{LiS}$ species, single $\mathrm{Li}^{+}$, and single electron, that is, $\mathrm{Li}_{2} \mathrm{~S} \rightarrow \mathrm{LiS}+$ $\mathrm{Li}^{+}+\mathrm{e}^{-}$, has been considered. During the reaction, $\mathrm{Li}^{+}$moves away from the $\mathrm{S}$ atom in the $\mathrm{Li}_{2} \mathrm{~S}$ molecule, by the breaking of the $\mathrm{Li}-\mathrm{S}$ bond. Figure 4 shows the decomposition energy barrier of $\mathrm{Li}_{2} \mathrm{~S}$ species on the surface of both the configuration of the polypeptoid material. The calculated energy barrier is found to be $1.51 \mathrm{eV}$ in conf.-2 which is lower than the graphene. ${ }^{56}$ It means that the polypeptoid material is a superior candidate for improving the performance of $\mathrm{Li}-\mathrm{S}$ batteries.

\section{CONCLUSIONS}

We have systematically investigated the polypeptoid material as an anchoring material for $\mathrm{Li}-\mathrm{S}$ batteries. From the firstprinciples calculations, we have calculated the binding energy of LiPS species on the surface of the polypeptoid material which are higher than the binding energy of the electrolytes with LiPS species. Additionally, the diffusion energy barrier of $\mathrm{Li}_{2} \mathrm{~S}$ for conf.- 1 is lower than the conf.- 2 of the polypeptoid material and the decomposition energy barrier is relatively low as compared to most of the materials used in $\mathrm{Li}-\mathrm{S}$ batteries. These findings results suggest that the polypeptoid material can display strong binding energy and fast diffusion, confirming the rapid discharge and charging processes for LiS batteries. The present work demonstrates that the polypeptoid material will be a good candidate for the sulfur cathode.

\section{ASSOCIATED CONTENT}

\section{SI Supporting Information}

The Supporting Information is available free of charge at https://pubs.acs.org/doi/10.1021/acsaem.1c02712.

AIMD simulated structures of Nte4-Npe6 and Nte4N4Brpe6 polypeptoid material, fully optimized structures of LiPSs, $\mathrm{S}_{8}$ species and organic electrolyte, side view of adsorbate species on the polypeptoid surface, and lowest energy configurations of LiPS species with organic electrolytes DOL and DME (PDF)

\section{AUTHOR INFORMATION}

\section{Corresponding Authors}

Deobrat Singh - Condensed Matter Theory Group, Materials Theory Division, Department of Physics and Astronomy, Uppsala University, 75120 Uppsala, Sweden; 이이.org/ 0000-0001-7246-8743; Email: deobrat.singh@ physics.uu.se

Rajeev Ahuja - Condensed Matter Theory Group, Materials Theory Division, Department of Physics and Astronomy, Uppsala University, 75120 Uppsala, Sweden; Department of Physics, Indian Institute of Technology Ropar, Rupnagar 140001 Punjab, India; 1 orcid.org/0000-0003-1231-9994; Email: rajeev.ahuja@physics.uu.se

Complete contact information is available at: https://pubs.acs.org/10.1021/acsaem.1c02712

\section{Notes}

The authors declare no competing financial interest.

\section{ACKNOWLEDGMENTS}

D.S. and R.A. thank the Swedish Research Council (VR-201606014 and VR2020-04410) and J. Gust. Richert stiftelse, Sweden (2021-00665) for financial support. D.S. and R.A. gratefully acknowledge computational resources from the Swedish National Infrastructure for Computing SNIC and HPC2N.

\section{REFERENCES}

(1) Liu, B.; Bo, R.; Taheri, M.; Di Bernardo, I.; Motta, N.; Chen, H.; Tsuzuki, T.; Yu, G.; Tricoli, A. Metal-Organic Frameworks/ Conducting Polymer Hydrogel Integrated Three-Dimensional FreeStanding Monoliths as Ultrahigh Loading Li-S Battery Electrodes. Nano Lett. 2019, 19, 4391-4399.

(2) Bai, L.; Chao, D.; Xing, P.; Tou, L. J.; Chen, Z.; Jana, A.; Shen, Z. X.; Zhao, Y. Refined Sulfur Nanoparticles Immobilized in MetalOrganic Polyhedron as Stable Cathodes for Li-S Battery. ACS Appl. Mater. Interfaces 2016, 8, 14328-14333.

(3) Li, M.; Wan, Y.; Huang, J.-K.; Assen, A. H.; Hsiung, C.-E.; Jiang, H.; Han, Y.; Eddaoudi, M.; Lai, Z.; Ming, J.; Li, L.-J. Metal-Organic Framework-Based Separators for Enhancing Li-S Battery Stability: Mechanism of Mitigating Polysulfide Diffusion. ACS Energy Lett. 2017, 2, 2362-2367.

(4) Gao, G.; Sun, X.; Wang, L.-W. An inverse vulcanized conductive polymer for Li-S battery cathodes. J. Mater. Chem. A 2020, 8, 2171121720.

(5) Zubi, G.; Dufo-López, R.; Carvalho, M.; Pasaoglu, G. The lithiumion battery: State of the art and future perspectives. Renewable Sustainable Energy Rev. 2018, 89, 292-308.

(6) Wu, H. B.; Wei, S.; Zhang, L.; Xu, R.; Hng, H. H.; Lou, X. W. D. Embedding Sulfur in MOF-Derived Microporous Carbon Polyhedrons for Lithium-Sulfur Batteries. Chem._Eur. J. 2013, 19, 10804-10808.

(7) Bai, L.; Tu, B.; Qi, Y.; Gao, Q.; Liu, D.; Liu, Z.; Zhao, L.; Li, Q.; Zhao, Y. Enhanced performance in gas adsorption and $\mathrm{Li}$ ion batteries by docking $\mathrm{Li}+$ in a crown ether-based metal-organic framework. Chem. Commun. 2016, 52, 3003-3006.

(8) Yang, X.; Li, X.; Adair, K.; Zhang, H.; Sun, X. Structural Design of Lithium-Sulfur Batteries: From Fundamental Research to Practical Application. Electrochem. Energy Rev. 2018, 1, 239-293.

(9) Zhang, Z.; Kong, L.-L.; Liu, S.; Li, G.-R.; Gao, X.-P. A HighEfficiency Sulfur/Carbon Composite Based on 3D Graphene Nanosheet@Carbon Nanotube Matrix as Cathode for Lithium-Sulfur Battery. Adv. Energy Mater. 2017, 7, 1602543.

(10) Mikhaylik, Y. V.; Akridge, J. R. Polysulfide shuttle study in the Li/ S battery system. J. Electrochem. Soc. 2004, 151, A1969.

(11) Zheng, G.; Yang, Y.; Cha, J. J.; Hong, S. S.; Cui, Y. Hollow carbon nanofiber-encapsulated sulfur cathodes for high specific capacity rechargeable lithium batteries. Nano Lett. 2011, 11, 4462-4467.

(12) Kolosnitsyn, V. S.; Karaseva, E. V. Lithium-sulfur batteries: Problems and solutions. Russ. J. Electrochem. 2008, 44, 506-509.

(13) Lin, Z.; Liu, Z.; Dudney, N. J.; Liang, C. Lithium Superionic Sulfide Cathode for All-Solid Lithium-Sulfur Batteries. ACS Nano 2013, 7, 2829-2833.

(14) Wang, J.; Si, L.; Wei, Q.; Hong, X.; Lin, L.; Li, X.; Chen, J.; Wen, P.; Cai, Y. An imine-linked covalent organic framework as the host material for sulfur loading in lithium-sulfur batteries. J. Energy Chem. 2019, 28, 54-60.

(15) Baumann, A. E.; Burns, D. A.; Díaz, J. C.; Thoi, V. S. Lithiated Defect Sites in Zr Metal-Organic Framework for Enhanced Sulfur Utilization in Li-S Batteries. ACS Appl. Mater. Interfaces 2018, 11, 2159-2167.

(16) Wang, H.; Yang, Y.; Liang, Y.; Robinson, J. T.; Li, Y.; Jackson, A.; Cui, Y.; Dai, H. Graphene-Wrapped Sulfur Particles as a Rechargeable Lithium-Sulfur Battery Cathode Material with High Capacity and Cycling Stability. Nano Lett. 2011, 11, 2644-2647.

(17) Chen, S.-R.; Zhai, Y.-P.; Xu, G.-L.; Jiang, Y.-X.; Zhao, D.-Y.; Li, J.T.; Huang, L.; Sun, S.-G. Ordered mesoporous carbon/sulfur 
nanocomposite of high performances as cathode for lithium-sulfur battery. Electrochim. Acta 2011, 56, 9549-9555.

(18) Navaneedhakrishnan, J.; Shen, J.; Moganty, S. S.; Corona, A.; Archer, L. Porous Hollow Carbon Sulfur Composites for High Power Lithium-Sulfur Batteries. ECS Meeting Abstracts; IOP Publishing, 2011; Vo. 341.

(19) Zheng, J.; Guo, G.; Li, H.; Wang, L.; Wang, B.; Yu, H.; Yan, Y.; Yang, D.; Dong, A. Elaborately Designed Micro-Mesoporous Graphitic Carbon Spheres as Efficient Polysulfide Reservoir for Lithium-Sulfur Batteries. ACS Energy Lett. 2017, 2, 1105-1114.

(20) Luo, L.; Manthiram, A. Rational Design of High-Loading Sulfur Cathodes with a Poached-Egg-Shaped Architecture for Long-Cycle Lithium-Sulfur Batteries. ACS Energy Lett. 2017, 2, 2205-2211.

(21) Kim, H. M.; Sun, H.-H.; Belharouak, I.; Manthiram, A.; Sun, Y.K. An Alternative Approach to Enhance the Performance of High Sulfur-Loading Electrodes for Li-S Batteries. ACS Energy Lett. 2016, 1, 136-141.

(22) Je, S. H.; Hwang, T. H.; Talapaneni, S. N.; Buyukcakir, O.; Kim, H. J.; Yu, J.-S.; Woo, S.-G.; Jang, M. C.; Son, B. K.; Coskun, A.; Choi, J. W. Rational Sulfur Cathode Design for Lithium-Sulfur Batteries: SulfurEmbedded Benzoxazine Polymers. ACS Energy Lett. 2016, 1, 566-572.

(23) Zhang, X.; Cheng, X.; Zhang, Q. Nanostructured energy materials for electrochemical energy conversion and storage: a review. J. Energy Chem. 2016, 25, 967-984.

(24) Peng, H.-J.; Huang, J.-Q.; Cheng, X.-B.; Zhang, Q. LithiumSulfur Batteries: Review on High-Loading and High-Energy LithiumSulfur Batteries (Adv. Energy Mater. 24/2017). Adv. Energy Mater. 2017, 7, 1770141 .

(25) Wu, F.; Chen, J.; Chen, R.; Wu, S.; Li, L.; Chen, S.; Zhao, T. Sulfur/polythiophene with a core/shell structure: synthesis and electrochemical properties of the cathode for rechargeable lithium batteries. J. Phys. Chem. C 2011, 115, 6057-6063.

(26) Li, G.-C.; Li, G.-R.; Ye, S.-H.; Gao, X.-P. A polyaniline-coated sulfur/carbon composite with an enhanced high-rate capability as a cathode material for lithium/sulfur batteries. Adv. Energy Mater. 2012, $2,1238-1245$.

(27) Xi, K.; Cao, S.; Peng, X.; Ducati, C.; Vasant Kumar, R.; Cheetham, A. K. Carbon with hierarchical pores from carbonized metal-organic frameworks for lithium sulphur batteries. Chem. Commun. 2013, 49, 2192-2194.

(28) Ghazi, Z. A.; He, X.; Khattak, A. M.; Khan, N. A.; Liang, B.; Iqbal, A.; Wang, J.; Sin, H.; Li, L.; Tang, Z. MoS2 /Celgard Separator as Efficient Polysulfide Barrier for Long-Life Lithium-Sulfur Batteries. Adv. Mater. 2017, 29, 1606817.

(29) Sun, J.; Sun, Y.; Pasta, M.; Zhou, G.; Li, Y.; Liu, W.; Xiong, F.; Cui, Y. Entrapment of Polysulfides by a Black-Phosphorus-Modified Separator for Lithium-Sulfur Batteries. Adv. Mater. 2016, 28, 97979803.

(30) Jewel, Y.; Yoo, K.; Liu, J.; Dutta, P. Self-assembled peptides for coating of active sulfur nanoparticles in lithium-sulfur battery. J. Nanoparticle Res. 2016, 18, 54.

(31) Xuan, S.; Jiang, X.; Spencer, R. K.; Li, N. K.; Prendergast, D.; Balsara, N. P.; Zuckermann, R. N. Atomic-level engineering and imaging of polypeptoid crystal lattices. Proc. Natl. Acad. Sci. 2019, 116, 22491-22499.

(32) Zhang, J.; Yang, G.; Tian, J.; Wang, Y.; Ma, D. Modulating electronic and optical properties of black phosphorous carbide monolayers by molecular doping. Appl. Surf. Sci. 2018, 448, 270-280.

(33) Kresse, G.; Joubert, D. From ultrasoft pseudopotentials to the projector augmented-wave method. Phys. Rev. B: Condens. Matter Mater. Phys. 1999, 59, 1758.

(34) Kresse, G.; Furthmüller, J. Efficiency of ab-initio total energy calculations for metals and semiconductors using a plane-wave basis set. Comput. Mater. Sci. 1996, 6, 15-50.

(35) Kresse, G.; Furthmüller, J. Efficient iterative schemes forab initiototal-energy calculations using a plane-wave basis set. Phys. Rev. B: Condens. Matter Mater. Phys. 1996, 54, 11169.

(36) Blöchl, P. E. Projector augmented-wave method. Phys. Rev. B: Condens. Matter Mater. Phys. 1994, 50, 17953.
(37) Perdew, J. P.; Burke, K.; Ernzerhof, M. Generalized gradient approximation made simple. Phys. Rev. Lett. 1996, 77, 3865.

(38) Grimme, S.; Antony, J.; Ehrlich, S.; Krieg, H. A consistent and accurate $\mathrm{ab}$ initio parametrization of density functional dispersion correction (DFT-D) for the 94 elements H-Pu. J. Chem. Phys. 2010, 132, 154104.

(39) Grimme, S.; Ehrlich, S.; Goerigk, L. Effect of the damping function in dispersion corrected density functional theory. J. Comput. Chem. 2011, 32, 1456-1465.

(40) Momma, K.; Izumi, F. VESTA: a three-dimensional visualization system for electronic and structural analysis. J. Appl. Crystallogr. 2008, $41,653-658$.

(41) Ruiz-Serrano, Á.; Hine, N. D. M.; Skylaris, C.-K. Pulay forces from localized orbitals optimized in situ using a psinc basis set. J. Chem. Phys. 2012, 136, 234101.

(42) Henkelman, G.; Jónsson, H. Improved tangent estimate in the nudged elastic band method for finding minimum energy paths and saddle points. J. Chem. Phys. 2000, 113, 9978-9985.

(43) Henkelman, G.; Uberuaga, B. P.; Jónsson, H. A climbing image nudged elastic band method for finding saddle points and minimum energy paths. J. Chem. Phys. 2000, 113, 9901-9904.

(44) Martyna, G. J.; Klein, M. L.; Tuckerman, M. Nosé-Hoover chains: The canonical ensemble via continuous dynamics. J. Chem. Phys. 1992, 97, 2635-2643.

(45) Henkelman, G.; Arnaldsson, A.; Jónsson, H. A fast and robust algorithm for Bader decomposition of charge density. Comput. Mater. Sci. 2006, 36, 354-360.

(46) Liu, W.; Carrasco, J.; Santra, B.; Michaelides, A.; Scheffler, M.; Tkatchenko, A. Benzene adsorbed on metals: Concerted effect of covalency and van der Waals bonding. Phys. Rev. B: Condens. Matter Mater. Phys. 2012, 86, 245405.

(47) Fang, M.; Liu, X.; Ren, J.-C.; Yang, S.; Su, G.; Fang, Q.; Lai, J.; Li, S.; Liu, W. Revisiting the anchoring behavior in lithium-sulfur batteries: many-body effect on the suppression of shuttle effect. npj Comput. Mater. 2020, 6, 8 .

(48) Manthiram, A.; Fu, Y.; Chung, S.-H.; Zu, C.; Su, Y.-S. Rechargeable Lithium-Sulfur Batteries. Chem. Rev. 2014, 114, 11751-11787.

(49) Mikhaylik, Y. V.; Kovalev, I.; Schock, R.; Kumaresan, K.; Xu, J.; Affinito, J. High energy rechargeable Li-S cells for EV application: status, remaining problems and solutions. ECS Trans. 2010, 25, 23.

(50) Fang, Q.; Fang, M.; Liu, X.; Yu, P.; Ren, J.-C.; Li, S.; Liu, W. An asymmetric $\mathrm{Ti} 2 \mathrm{CO} / \mathrm{WS} 2$ heterostructure as a promising anchoring material for lithium-sulfur batteries. J. Mater. Chem. A 2020, 8, 1377013775 .

(51) Zhang, Q.; Wang, Y.; Seh, Z. W.; Fu, Z.; Zhang, R.; Cui, Y. Understanding the Anchoring Effect of Two-Dimensional Layered Materials for Lithium-Sulfur Batteries. Nano Lett. 2015, 15, 37803786.

(52) Zhao, J.; Yang, Y.; Katiyar, R. S.; Chen, Z. Phosphorene as a promising anchoring material for lithium-sulfur batteries: a computational study. J. Mater. Chem. A 2016, 4, 6124-6130.

(53) Song, X.; Qu, Y.; Zhao, L.; Zhao, M. Monolayer Fe3GeX2 (X = S, $\mathrm{Se}$, and $\mathrm{Te})$ as Highly Efficient Electrocatalysts for Lithium-Sulfur Batteries. ACS Appl. Mater. Interfaces 2021, 13, 11845-11851.

(54) Tao, X.; Wang, J.; Liu, C.; Wang, H.; Yao, H.; Zheng, G.; Seh, Z. W.; Cai, Q.; Li, W.; Zhou, G.; Zu, C.; Cui, Y. Balancing surface adsorption and diffusion of lithium-polysulfides on nonconductive oxides for lithium-sulfur battery design. Nat. Commun. 2016, 7, 11203.

(55) Jing, Y.; Zhou, Z.; Cabrera, C. R.; Chen, Z. Metallic VS2 monolayer: a promising $2 \mathrm{D}$ anode material for lithium ion batteries. $J$. Phys. Chem. C 2013, 117, 25409-25413.

(56) Zhou, G.; Tian, H.; Jin, Y.; Tao, X.; Liu, B.; Zhang, R.; Seh, Z. W.; Zhuo, D.; Liu, Y.; Sun, J.; Zhao, J.; Zu, C.; Wu, D. S.; Zhang, Q.; Cui, Y. Catalytic oxidation of $\mathrm{Li} 2 \mathrm{~S}$ on the surface of metal sulfides for $\mathrm{Li}-\mathrm{S}$ batteries. Proc. Natl. Acad. Sci. 2017, 114, 840-845. 